\title{
A Hybrid Range-Free Algorithm Using Dynamic Communication Range for Wireless Sensor Networks
}

https://doi.org/10.3991/ijoe.v16i08.14379

\author{
Fengrong Han $\left({ }^{\bowtie}\right)$, Izzeldin Ibrahim Mohamed Abdelaziz, \\ Xinni Liu, Kamarul Hawari Ghazali \\ University Malaysia Pahang, Pekan, Malaysia \\ hfr825@163. com \\ Hao Wang \\ University Malaysia Pahang, Gambang, Malaysia
}

\begin{abstract}
Location plays a backbone role in networks, since it will great influence basic wireless sensor networks (WSNs) architecture. Distance-Vector Hop (DV-Hop) is a representative range-free localization algorithm, which is widely utilized to locate node position in location-based application. However, with poor localization accuracy, it cannot satisfy precise location-based application requirement. Consequently, we proposed a hybrid range-free algorithm depends on dynamic communication range to address low localization accuracy problem, named as DCDV-Hop. Firstly, we applied statistical methods to analyze the relationship between location error and hop count under different communication ranges. Thereafter, we employed centroid algorithm to calculate target node coordinate based on hop threshold. Finally, a weighted least square is applied to locate remaining target nodes. We conducted considerable experiments, the results demonstrated that our proposed algorithm DCDV-Hop can effectively reduce accumulate localization error and improve localization accuracy of target nodes, with stable performance. Moreover, maximum localization accuracy reached up to $90.94 \%$ and localization error reduced more than $50 \%$, compared with DV-Hop algorithm.
\end{abstract}

Keywords-WSNs, Range-free localization, DV-Hop, Communication range, Anchor node.

\section{Introduction}

Advanced electrical sensor chips and short-range wireless communication technologies have foster numerous location-based applications, it as spring bamboo shoots poured into people daily lives in past two decades. Wireless sensor networks (WSNs) are connection bridge between physical world and digital world, which is wildly adopted in diverse applications, such as animal tracking, forest or flood monitoring, military reconnaissance, and even energy management [1]. Sensor node is composed of wireless communication module, sensor module, control module, storage module, and computing module. Sensor node transmitted collected information of physical 
world to monitoring center or user. If perception data without position information, it will be lost meaning, specifically for precise location-based applications. Therefore, localization technique has developed into one of the most fundamental issues for WSNs [2, 3].

According to whether position is known or not, sensors nodes are divided into two categories, one is called as beacon node or anchor node, the other one is called as target nodes or unknown nodes. Anchor node is with fixed location, that manually deployed or attached with navigation equipment, like GPS. Cost of anchor noes is larger than normal nodes due to attached navigation equipment, and so the number of anchor node utilized in sensor network is small. Target node is randomly distributed in monitor area by many deploy methods, such as aircraft scatter [3]. In wireless sensor network, target nodes relied on anchor nodes to locate their position, when target nodes received location information form more than three anchor nodes, its coordinate can be estimated by using trilateration, multilateral measurement etc. [4].

Currently, proposed localization schemes are broadly divided into range-based and range-free positioning algorithms based on whether attached Global Positioning System (GPS) chips on sensor to range angle and distance for location positioning. Range-based scheme estimated target sensor location based on perceived distance or angle, it can obtain high localization accuracy. However, it not only required complex hardware circuits but also consumed high energy. That is mainly including Angle of Arrival (AOA) [5], Time of Arrival (TOA) [6], Time Difference of Arrival (TDOA) [6] and Received Signal Strength Indicator (RSSI) [7].

Due to limited battery and overhead energy, range-free localization scheme is more practical and popular for its simplicity. Currently, range-free algorithm mainly included Centroid [8], Amorphous [9], multidimensional scaling (MDS) [10], DV-Hop (Distance-Vector-Hop) [11], and Approximate Point in Triangle (APIT) [12] etc. DVHop is one of classical range-free algorithms, it estimated distance between anchor and target node based on hop counts. DV-Hop can obtain high located ratio even in low network density. However, due to average hop size is utilized instead of linear distance, its localization accuracy is easily affected by network topology and some nodes got high localization error.

Aimed at enhancing localization accuracy for DV-Hop algorithm, we proposed a hybrid range-free algorithm based on weighted dynamic communication range for WSNs to address poor location accuracy problem. Firstly, we applied statistical methods to analyze the relationship between location error and hop count under different communication ranges. Then, we employed dynamic communication range based on hop counts to narrow hop size error. Finally, improved centroid algorithm and weighted least squares are adopted to estimate target node coordinate according to hop threshold.

The remaining paper is formulated as following. Current representatives research works are summarized and evaluated in part 2. Basic DV-Hop algorithm work flow is introduced in part 3. Part 4 presented detail information of our proposed enhanced algorithm (WDCDV-Hop). Extensive experiment and conclusions are illustrated in part 5 and part 6 , respectively. 


\section{Related Works}

Multiple scholars have proposed corresponding improved localization algorithms from various aspects to overcome shortcomings of DV-Hop. Shi et al [13] employed RSSI technology to calculate hop counts between anchor nodes. Quasi-Newton optimization method is applied to estimate location coordinate of target node in third phase. However, it will increase hardware cost since introduced RSSI. Minimum hop count is optimized by employed RSSI technology in reference [14]. In addition, average hop size is controlled by anchor node proportion. Wu et al. introduced a new localization method based on distance-assisted (LDA) in paper [15], which introduced curving fitting method in third phase to calculate location coordinate. Simulation results presented good performance in accuracy. Two corrections are weighed for average hop size in reference [16]. It is given higher weighted coefficient for one hop count of target node. Li et al. designed a newly 3D location algorithm in paper [17]. Firstly, average hop distance is calculation by weighted coefficient. The anchor node is selected based on topology. A weighted centroid algorithm is utilized to estimate the coordinate of target node, which greatly reduced computational complexity and localization error. A modified hop size is presented in reference [18], which is based on actual distance between anchor nodes. In addition, max received value of anchor node is utilized to instead of the first received one.

Zheng et al. proposed an improved localization (DV-SCA) [19]. Firstly, average hop distance is correct by actual distance of anchor node. Then, optimal anchor nodes are selected by APIT algorithm, which depended on whether unknown node is in acute triangle or not. If increased anchor node ratio, it can get a higher positioning accuracy. However, that increased hardware cost. To reduce cost, an empirical parameter as threshold value is set in paper [20]. The concept of proportional parameter is introduced to narrow average hop size in paper [21]. Simulation results shown that localization accuracy improved $10.2 \%$, comparing with traditional DV-Hop. CAO et al. [22] proposed a novel DV-Hop algorithm, named as (DANS IDV-Hop). A dynamic anchor node set is utilized to select suitable anchor nodes for target node, which transformed location issue to be combinational optimization problem. Then, binary particle swarm optimization is employed to structure dynamic anchor node set. Lastly, continuous particle swarm optimization is proposed to further correct target node position. However, DANS IDV-Hop increased location time for iteration times. A PSO-QNN model is proposed by Liu et al [23] in DV-Hop which combined particle swarm optimization and quantum neural network. Levenberg Marquardt method is applied in third phase of DV-Hop to instead of least squares in paper [24]. A hybrid based on RSSI and PSO algorithm is proposed in [25], however, it increased computational complexity.

There are still many drawbacks in current improved DV-Hop algorithm, such as smaller increased accuracy, complex algorithm and excessive overhead energy consumption etc. We introduced our upgrade algorithm to address high localization error issue. 


\section{DV-Hop Algorithm and Error Analysis}

\subsection{DV-Hop algorithm model}

The DV-Hop localization algorithm is developed by Dragos Niculescu et al. for distributed networks. The localization process consisted of 3 basic steps.

Step 1: Estimate minimum hop counts between nodes: All anchor nodes send packet information with position and hop count data to neighbour node, after communicable node received data, if precious hop count is larger, data hop count plus 1 . Then, forward to target node that has not obtained data packet, until whole network got minimum hops between nodes.

Here, Fig. 1 is an illustration of DV-Hop algorithm, $A_{l}, A_{2}$ and $A_{3}$ are anchor nodes, and the rest nodes are target nodes. It is assumed target node $T_{i}$ is the one that needed to be located. From the first step, we can get minimum hop $A_{1}$ to $A_{2}, A_{2}$ to $A_{3}$ and $A_{1}$ to $A_{3}$ is 2,5 and 7 , respectively. The minimum hop $T_{i}$ to $A_{1}, A_{2}$ and $A_{3}$ is 4,2 and 3 , respectively.

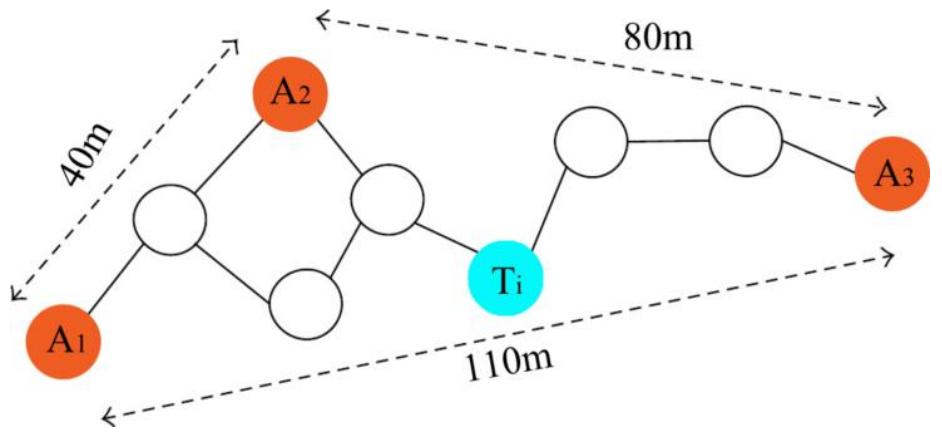

Fig. 1. An illustration of DV-Hop

Step2: Estimate average hop size: All anchor nodes obtained minimum hop value and node location information after Step 1. It adopted Equation (1) to calculate average hop size.

$$
\operatorname{AvgHopSize}_{i}=\frac{\Sigma_{i \neq j}^{m} \sqrt{\left(x_{i}-x_{j}\right)^{2}+\left(y_{i}-y_{j}\right)^{2}}}{\Sigma_{i \neq j}^{m} H_{i j}}
$$

Where, $\left(x_{i}, y_{i}\right)$ and $\left(x_{j}, y_{j}\right)$ are the coordinate of anchor node $i$ and $j$, respectively. $H_{i j}$ is hop counts between anchor node $i$ and $j$. AvgHopSize $e_{i}$ denoted average hop size of anchor node $i$.

Taken Fig. 1 as an example, average hop size of $A_{1}, A_{2}$ and $A_{3}$ can be calculated as following.

AvgHopSize_A $A_{1}=(40+110) /(2+7)=16.67$
AvgHopSize_A $A_{2}=(40+80) /(2+5)=17.14$
AvgHopSize_A $A_{3}=(110+80) /(7+5)=15.83$ 
Equation (2) is employed to calculate estimate distance dit between anchor node $\mathrm{i}$ and target node $\mathrm{t}$.

$$
d_{i t}=\text { AvgHopSize }_{i} \times H_{i t}
$$

Here, we still employed Fig. 1 as an instance. Since minimum hop of $T_{i}$ to $A_{1}, A_{2}$ and $A_{3}$ are 4, 2 and 3, respectively, so it chose AvgHopSize_A $A_{2}$ as target hop size to estimate the distance $T_{i}$ to $A_{1}, A_{2}$ and $A_{3}$, it can be obtained by Equation (2).

$$
\begin{aligned}
& \mathrm{d} \_A_{l_{-}} T_{i}=17.14 \times 4=68.56 \\
& \mathrm{~d} \_A_{2} T_{i}=17.14 \times 2=32.48 \\
& \mathrm{~d} \_A_{3} T_{i}=17.14 \times 3=51.42
\end{aligned}
$$

Step 3: Estimate target node coordinate: When target node got at least three estimated distance form anchor nodes, coordinates of target node can be estimated by maximum likelihood method or multilateral measurement method.

Let $\left(x_{t}, y_{t}\right)$ be coordinate of target node $t$, and $d_{i t}$ is distance between target node $t$ and anchor node $A_{i}, i \in\{1,2,3 \ldots \mathrm{n}\}, d_{i t}$ can be obtained based on Equation (2).

$$
\begin{gathered}
\left(x_{t}-x_{1}\right)^{2}+\left(y_{t}-y_{1}\right)^{2}=d_{1 t}^{2} \\
\left(x_{t}-x_{2}\right)^{2}+\left(y_{t}-y_{2}\right)^{2}=d_{2 t}^{2} \\
\cdot \\
\cdot \\
\left(x_{t}-x_{n}\right)^{2}+\left(y_{t}-y_{n}\right)^{2}=d_{n t}^{2}
\end{gathered}
$$

Equation (3) can be formulated into $\mathrm{AX}=\mathrm{B}$, see as follow.

$$
\begin{gathered}
A=-2 \times\left[\begin{array}{cc}
x_{1}-x_{n} & y_{1}-y_{n} \\
x_{2}-x_{n} & y_{2}-y_{n} \\
& \cdot \\
x_{n-1}-x_{n} & y_{n-1}-y_{n}
\end{array}\right] \\
X=\left[\begin{array}{l}
x_{u} \\
y_{u}
\end{array}\right] \\
B=\left[\begin{array}{c}
d_{1}^{2}-d_{n}^{2}-x_{1}^{2}+x_{n}^{2}-y_{1}^{2}+y_{n}^{2} \\
d_{2}^{2}-d_{n}^{2}-x_{2}^{2}+x_{n}^{2}-y_{2}^{2}+y_{n}^{2} \\
\cdot \\
\cdot \\
d_{n-1}^{2}-d_{n}^{2}-x_{n-1}^{2}+x_{n}^{2}-y_{n-1}^{2}+y_{n}^{2}
\end{array}\right]
\end{gathered}
$$

The coordinate $\left(x_{t}, y_{t}\right)$ of target node can be estimated by Equation (7).

$$
X=\left(A^{T} A\right)^{-1} A^{T} B
$$

\subsection{Localization error analysis}

The localization accuracy of DV-Hop algorithm is largely depended on whether estimated average hop size is reasonable. Employing one average hop size from nearest 
anchor node cannot fully represent the whole entire network. Especially, in multi-hop networks, since estimated distance between target node anchor node is not straight line, which will accumulate hop size error in lower density area. Hence, network connectivity and hop size between anchor nodes are two essential factors in DV-Hop localization algorithm. Moreover, communication range of anchor node is greatly affected average hop size and hop count between nodes.

In order to investigate the relationship of location error between communication radius and hop count, following experiment has conducted in this article. In this experiment, there are 100 anchor nodes random distributed in $100 \mathrm{~m} \times 100 \mathrm{~m}$ area, including 20 anchor nodes, communication range is $25 \mathrm{~m}, 30 \mathrm{~m}$ and $35 \mathrm{~m}$, respectively. The relationship between hop count and localization error of DV-Hop algorithm under different communication range is illustrated in Fig.2 and Fig.3.

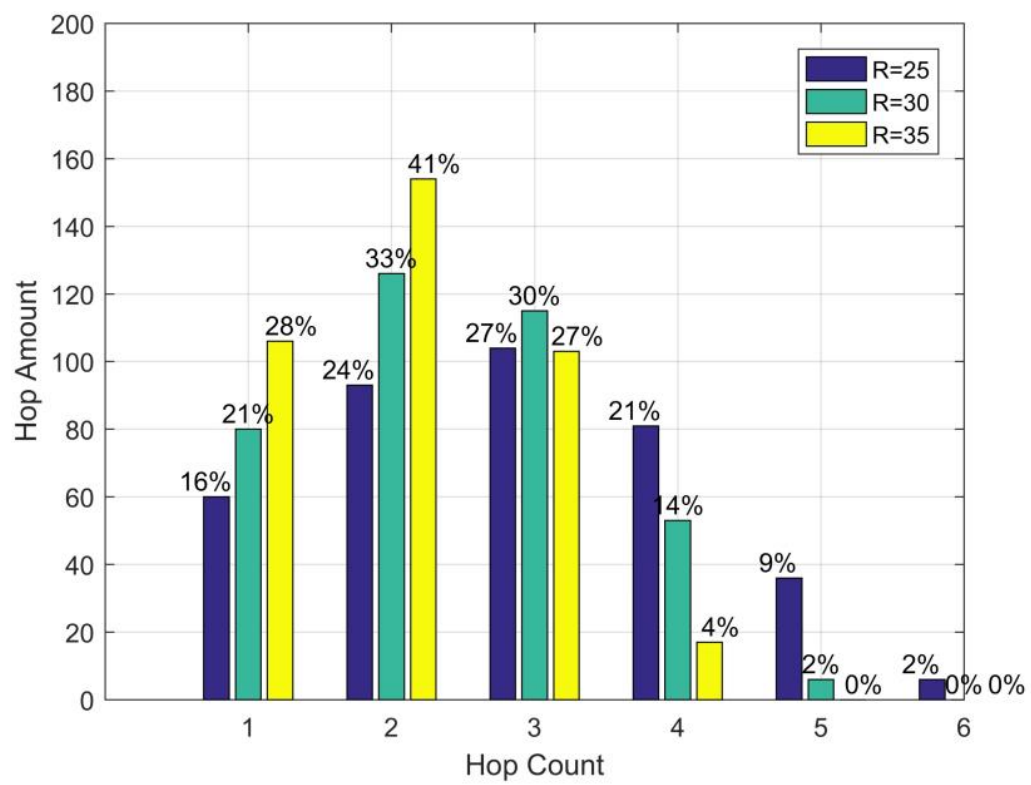

Fig. 2. Hop count under communication range $=25 \mathrm{~m}, 30 \mathrm{~m}, 35 \mathrm{~m}$

It is observed from Fig. 2 that hop count shown normal distribution trends under different communication ranges. The maximum hop count is 6,5 and 4 , under communication range $25 \mathrm{~m}, 30 \mathrm{~m}$ and $35 \mathrm{~m}$, respectively. Hop count 1, 4, 5 and 6 present decline trends with increasing communication range. Hop count value 2 is always the largest amount under communication range $30 \mathrm{~m}$ and $35 \mathrm{~m}$, respectively. Hop count value 2 is the greatest amount under communication range $25 \mathrm{~m}$.

It is illustrated in Fig. 3 that hop size error presents upward trend with increasing communication range, except hop count 1 . Hop count 2 with the lowest hop size error under various communication ranges. One hop count exhibits downward trend, that means, one hop with larger hop size error under larger communication. Hence, it is 
essential to correct hop count based on communication range, since it will greatly be affected hop count amount. So, if got relatively accurate hop distance, it will undoubtedly improve the localization accuracy of all target nodes. We introduced dynamic communication ranges to each anchor node, one is the same network communication range $R$, the other is $R / 2$. The hop count is no longer an integer number after introduced communication range $\mathrm{R} / 2$, which will greatly reduce hop size error of anchor nodes with one hop count.

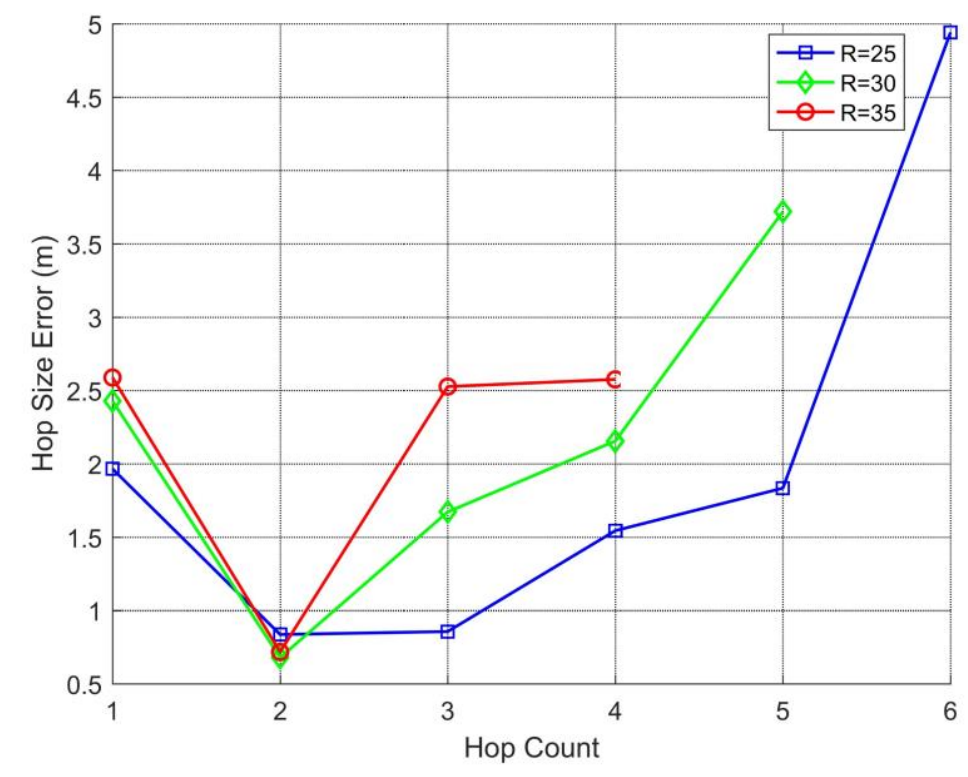

Fig. 3. Hop size error under communication range $=25 \mathrm{~m}, 30 \mathrm{~m}, 35 \mathrm{~m}$

\subsection{Verification based on dynamic communication ranges}

To investigate the effect of dynamic communication range on hop size, below experiment is conducted. It is assumed that there are 100 nodes dispersed in $100 \mathrm{~m} \times 100 \mathrm{~m}$ area, included 20 beacon nodes and communication radius is $25 \mathrm{~m}$. Hop size error is sharply reduced after adopted dynamic communication range that be illustrated in Fig. 4. 


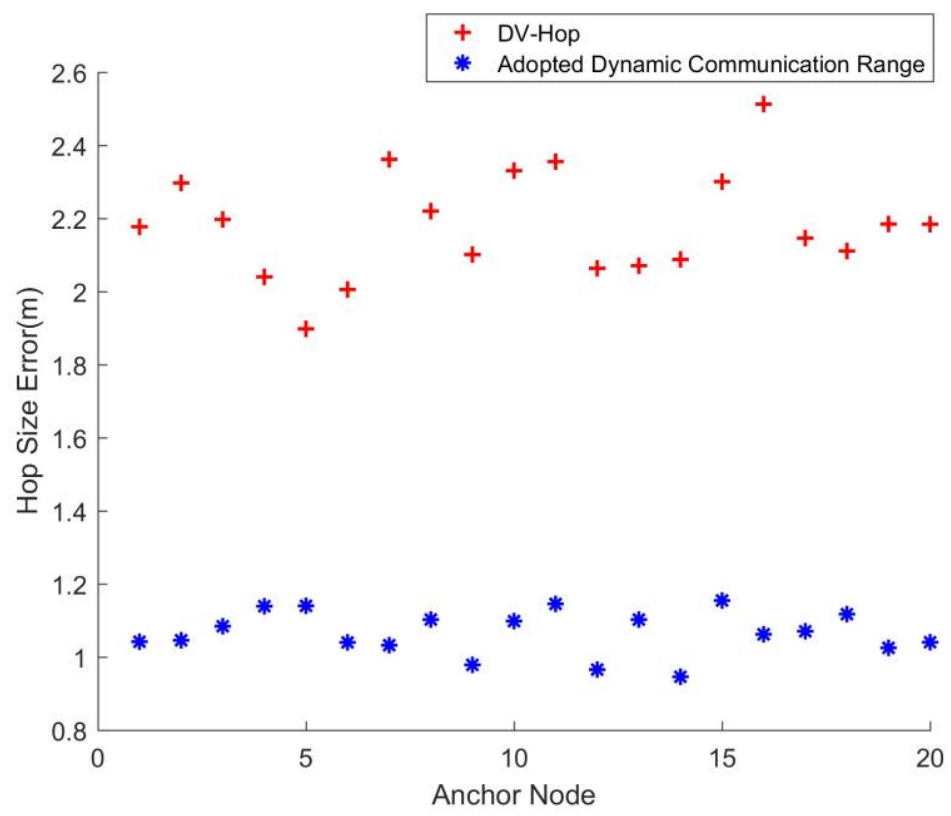

Fig. 4. Hop size error of anchor nodes

It is shown in Fig.4 that hop size error is around $1 \mathrm{~m}$ after applied dynamic communication range. And it is worth to point that hop size error is smooth with little fluctuation, that means, estimate distance error is small. However, hop size error of previous DV-Hop is around $2.2 \mathrm{~m}$, exceeded two times larger than employed dynamic communication range. Furthermore, hop size error is not stable, with larger fluctuation. In addition, the minimum hop size error of former DV-Hop is extremely bigger than introduced dynamic communication range, even compared with maximum error. Consequently, the verification confirmed feasibility of this method by introduced dynamic communication

\section{Proposed Algorithm (DCDV-Hop)}

\subsection{Hop counts correction}

It is assumed that target communication range is settled, but anchor node has two communication ranges, $\mathrm{R}$ and $\mathrm{R} / 2$. The implementation steps are listed as follows.

Step 1: Firstly, anchor node broadcasts information packet to whole network by $0.5 \mathrm{R}$ at first time, and its initial hop is 0 . After received information packet, communicable neighbor node updated the minimum hop count by 0.5 . In order to reduce communication overhead, the communicable node will not forward in the first broadcast. 
Step 2: After T times, anchor node broadcasts its information packet to the whole network for the second time with communication radius $\mathrm{R}$.

Step 3: If it is the first time of node received information packet from an anchor node, its hop count will be assigned to 1. Otherwise, compared it with previous hop count, keep smaller one. Add 1 to the stored hop count for continuing broadcasting.

As we all known, forward information will consume a large amount of communication energy during every flood. Due to each node has limited energy, hence, only second broadcast by flooding control. The first broadcast only sends information packet to communicable neighbor node. Since, the first broadcast with communication radius of $0.5 \mathrm{R}$, so, the minimum hop count is no longer integer.

\subsection{Average hop size correction}

It is can be obtained estimated distance between anchor node $i$ and $j$ using Equation (2). The error between actual distance and estimate distance denoted as $E_{i}$, which can obtain by Equation (10).

$$
E_{i}=\frac{1}{N-1} \sum_{i \neq j}\left(\sqrt{\left(x_{i}-x_{j}\right)^{2}+\left(y_{i}-y_{j}\right)^{2}}-\text { AvgHopSize }_{i} \times H_{i j}\right)
$$

Based on minimum mean-square error, let Equation (8) transformed into Equation (9).

$$
f=\frac{1}{N-1} \sum_{i \neq j}\left(\sqrt{\left(x_{i}-x_{j}\right)^{2}+\left(y_{i}-y_{j}\right)^{2}}-\text { AvgHopSize }_{i} \times H_{i j}\right)^{2}
$$

According to first derivative, let $\partial f / \partial$ AvgHopSize $_{i}=0$, we can get new equation to calculate average hop size.

$$
\operatorname{AvgHopSize}_{i}^{\text {New }}=\frac{\sum_{i \neq j}\left(H_{i j} \sqrt{\left(x_{i}-x_{j}\right)^{2}+\left(y_{i}-y_{j}\right)^{2}}\right)}{\sum_{i \neq j} H_{i j}^{2}}
$$

\subsection{Target node localization}

Let denoted actual coordinate of target node $X_{t}$, as $\left(x_{t}, y_{t}\right)$, distance between each anchor node $A_{k}(k=1,2,3 \ldots, N)$, is $d_{t k}$, which can be calculated by Equation (2).

1. Estimate target node coordinate based on hop threshold: It is observed from Fig. 2, the minimum hop size error is at hop count 2. It means less localization error. If target node received the number of anchor nodes $(m)$ with two hop counts that equal or more than three, these anchor nodes are randomly combined into $C_{m}^{3}$ triangles. Each triangle employed centroid algorithm to get estimated coordinate of target node. To get a better accuracy, re-employ coordinate algorithm on each obtained estimated coordinate. 


$$
X_{t}^{e}=\left(\frac{\Sigma_{t=1}^{C_{m}^{3}} x_{t}^{\prime}}{C_{m}^{3}}, \frac{\sum_{t=1}^{C_{m}^{3}} y_{t}^{\prime}}{C_{m}^{3}}\right)
$$

2. Estimate target node coordinate based on weighted least squares: Yan et al [26]. proposed an optimal weighted least square for irregular network. Based on this idea, we introduced it to regular network, employed a weighted coefficient matrix $\mathrm{W}$, see as following.

$$
W=\left[\begin{array}{cccc}
W_{1} & 0 & 0 & 0 \\
0 & W_{2} & 0 & 0 \\
. & . & . & . \\
. & . & . & . \\
0 & 0 & 0 & W_{k}
\end{array}\right]
$$

Where, $W_{k}=1 / H_{k}^{3}, H_{k}^{3}$ is the minimum number hop count between target node $X$ and anchor node $A$. Hence, Equation (9) can be transformed into Equation (13).

$$
X=\left(A^{T} W^{T} W A\right)^{-1} A^{T} W^{T} W B
$$

The flow chart of our proposed algorithm DCDV-Hop is illustrated in Fig. 5.

\section{$5 \quad$ Experimental Results and Discussion}

To verify the performance of our proposed algorithm DCDV-Hop, traditional DVHop localization algorithm is employed as compared in MATLAB 2016a. Parameters of experiment are listed in Table 1. A classical instance of distributed node is illustrated in Fig. 6, there are 100 nodes including 20 anchor nodes randomly deployed in 2D $100 \mathrm{~m} \times 100 \mathrm{~m}$ area. 


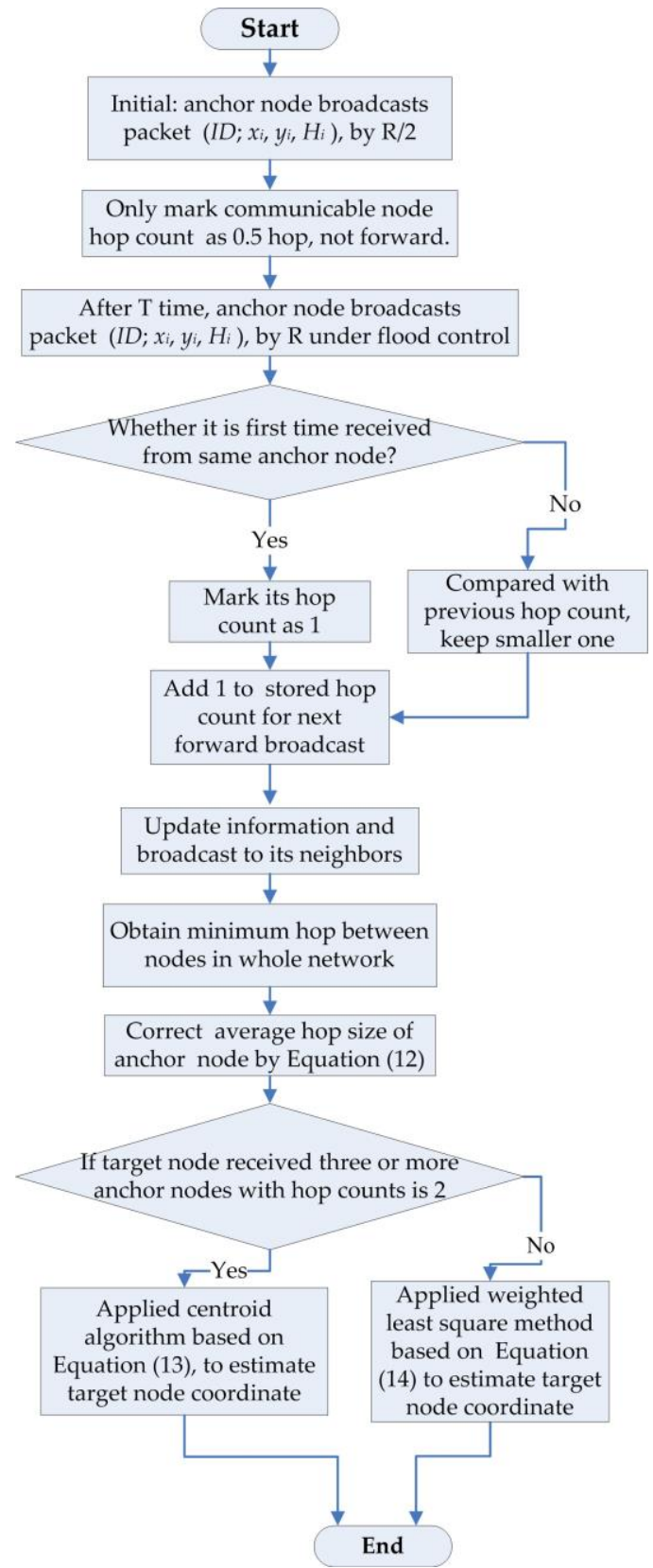

Fig. 5. The flow chart of DCDV-Hop 


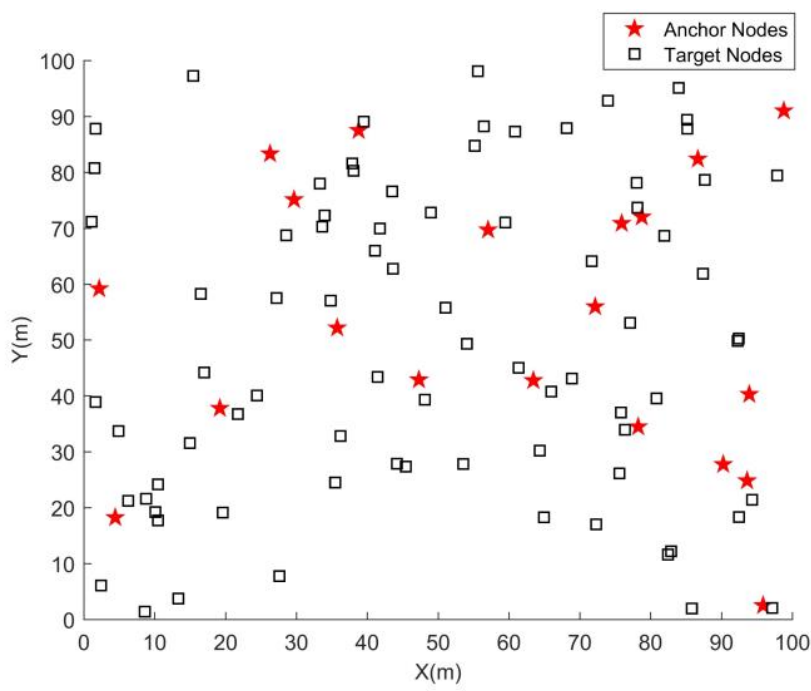

Fig. 6. A classic instance of distributed node

Table 1. The parameters setting for simulations

\begin{tabular}{|l|c|}
\hline \multicolumn{1}{|c|}{ Parameters } & Value \\
\hline Network area & $100 \mathrm{~m} \times 100 \mathrm{~m}$ \\
\hline Total nodes & $50-300$ \\
\hline Anchor nodes & $10-40$ \\
\hline Communication range & $20 \mathrm{~m}-36 \mathrm{~m}$ \\
\hline
\end{tabular}

\subsection{Performance evaluation criteria}

3. Localization error (LE): Localization error (LE) is the error between real and estimated coordinates of target node, which is denoted by Equation (16).

$$
L E=\sqrt{\left(x_{a}-x_{e}\right)^{2}+\left(y_{a}-y_{e}\right)^{2}}
$$

4. Average Localization Error (ALE): Average Localization Error (ALE) is average error of all target nodes.

$$
A L E=\frac{\sum_{t, a=1}^{n} \sqrt{\left(x_{a}-x_{e}\right)^{2}+\left(y_{a}-y_{e}\right)^{2}}}{n}
$$

5. Localization Accuracy (LA): Localization accuracy (LA) is defined as following.

$$
L A=\left(1-\frac{\sum_{u, a=1}^{n} \sqrt{\left(x_{a}-x_{e}\right)^{2}+\left(y_{a}-y_{e}\right)^{2}}}{n * R}\right) * 100 \%
$$

Here, $n$ represents total number of target nodes, $\left(x_{e}, y_{e}\right)$ and $\left(x_{a}, y_{a}\right)$ denotes estimated coordinate and actual coordinate, respectively. The communication range is $\mathrm{R}$. 


\subsection{Experiment results}

Experiments for all algorithms are conducted for 200 times for each result due to random deployment of nodes, adopted average value to evaluate the performance. We employed average LE, ALE and LA to evaluate the performance of our presented algorithm (DCDV-Hop).

1. Localization error for each target node: In this experiment, it is assumed that 100 senor nodes, involved 20 anchor nodes are randomly deployed in $100 \mathrm{~m} \times 100 \mathrm{~m}$ area. The sensor communication range is $20 \mathrm{~m}$. The LE for each unknown node is illustrated in Fig. 7 and Fig. 8.

Table 2. The localization error for each target node

\begin{tabular}{|l|c|c|c|c|}
\hline \multicolumn{1}{|c|}{ Localization Algorithm } & Max. LE(m) & Avg. LE(m) & Min. LE(m) & Std.LE(m) \\
\hline DV-Hop & 9.8358 & 8.4301 & 7.1207 & 0.5589 \\
\hline DCDV-Hop & 4.6500 & 3.9713 & 3.3182 & 0.1994 \\
\hline
\end{tabular}

The maximum LE, average LE, minimum LE and standard deviation of LE is illustrated in Table 2. It is observed form Table 2, the minimum LE of our proposed algorithm (DCDV-Hop) is less than $3.5 \mathrm{~m}$, with $53.40 \%$ decreased compared with DVHop algorithm, which stranded for it has a better performance. The max. LE and avg. LE are reduced $52.56 \%$ and $52.89 \%$, respectively. Moreover, standard deviation LE of DCDV-Hop is almost 3 times less than DV-Hop, that indicated DCDV-Hop is more stable.

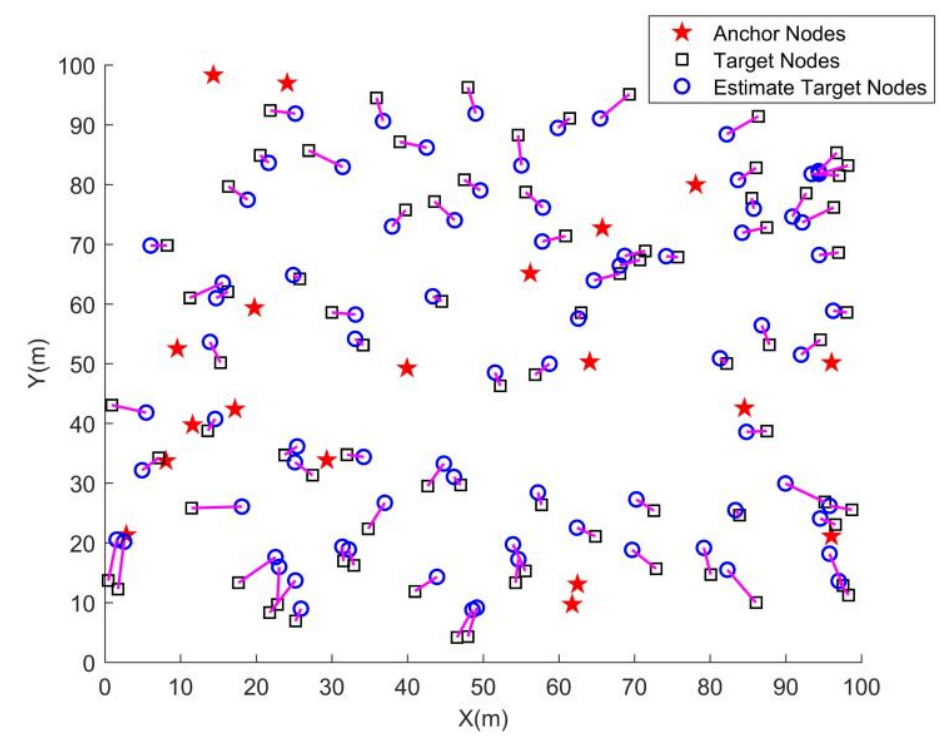

Fig. 7. The line of estimate and actual coordinate for target nodes 
It is illustrated in Fig. 7 that the line of estimate and actual coordinate for most target nodes is extremely short. It means that estimate coordinate of target node close to actual coordinate. There are only 12 target nodes cannot be located during 200 times, it stands for that located ratio is nearly $100 \%$.

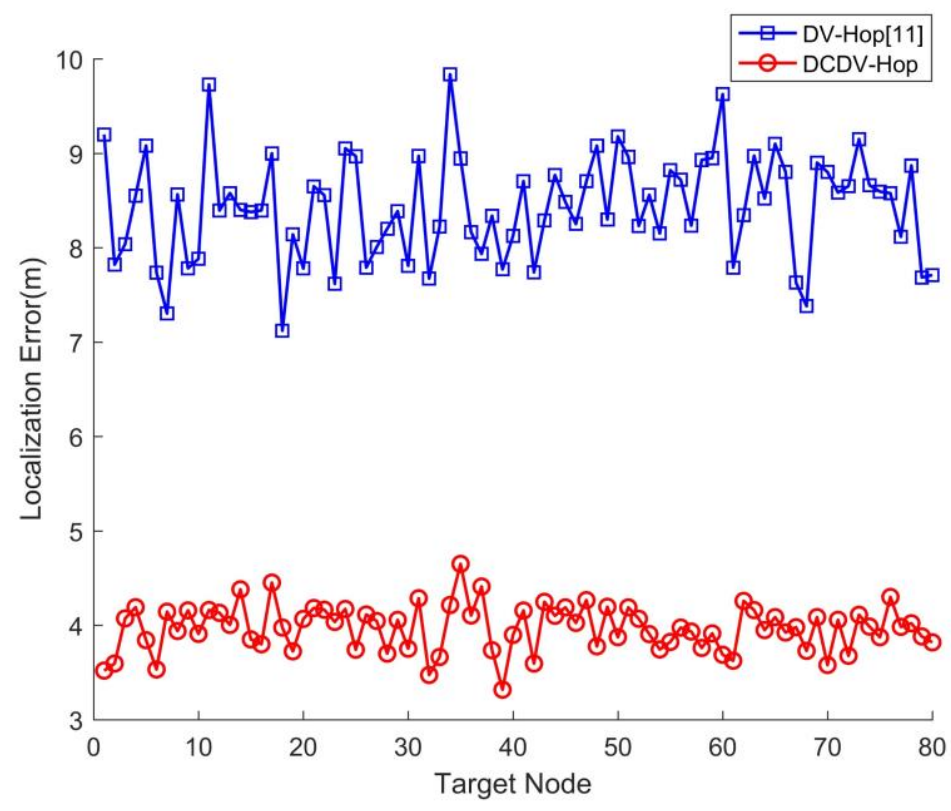

Fig. 8. The localization error for each target node

It can be seen from Fig. 8, the LE of basic DV-Hop algorithm is round 7-10m and our proposed algorithm (DCDV-Hop) is round $3-4 \mathrm{~m}$. Based on this figure, DCDVHop has a superior performance. The localization error of our proposed algorithm (DCDV-Hop) decreased about 55\%, compared with DV-Hop algorithm.

2. Effect of anchor nodes on algorithm: The experiment is conducted with 100 nodes that distributed in $100 \mathrm{~m} \times 100 \mathrm{~m}$ area. The communication range is $25 \mathrm{~m}$ and initial anchor node is 15 , with fixed 5 increased. The ALE and LA under different anchor node are presented in Fig. 9 and Fig.10 The maximum ALE and LA, average ALE and LA, minimum ALE and LA are demonstrated in Table 3. 


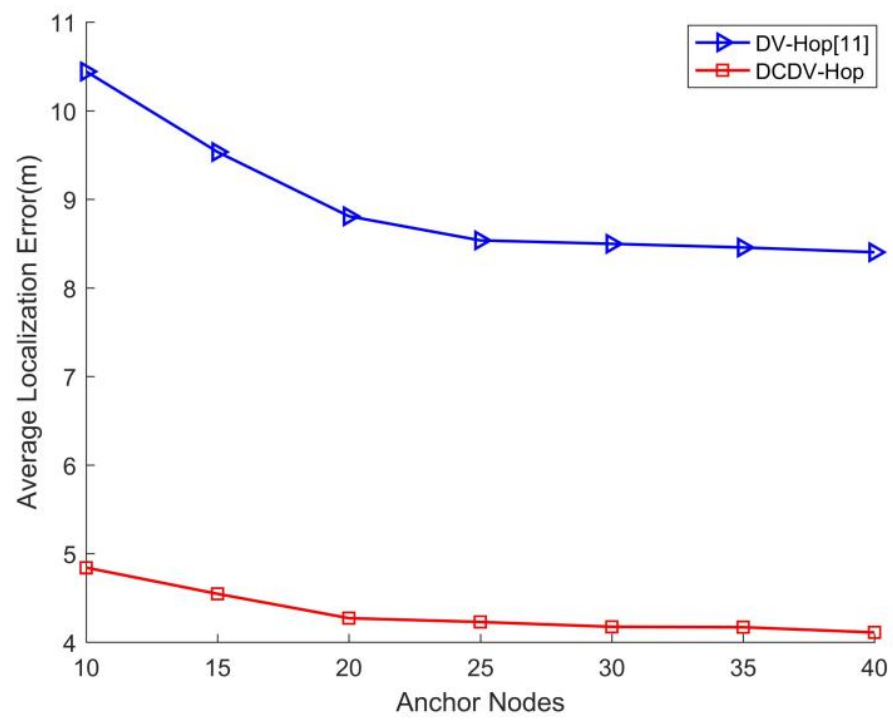

Fig. 9. The ALE under various anchor nodes

It can be seen form Fig.9 that the ALE of our proposed algorithm (DCDV-Hop) is less than 5, it reduced more 55\%, compared with DV-Hop. Moreover, the ALE is improved as the number of anchor nodes increases, whether it is a traditional algorithm DV-Hop or DCDV-Hop. When the proportion of anchor nodes increased from $10 \%$ to $20 \%$ in the network, the ALE curves of two algorithms have significantly decreased. The ALE curves of the two algorithms decrease slowly, when the proportion of anchor nodes increases from $20 \%$ to $40 \%$. When anchor nodes ratio is larger than $20 \%$, as anchor nodes density increased, the ALE of both algorithms tended to stabilize, and anchor node density no longer has large effect on ALE.

As it is depicted in Fig.10, the LA of two algorithms demonstrated upward trend, as anchor node increased. Both algorithms present stable tendency after anchor node ratio large than $20 \%$. The LA of our propose algorithm DCDV-Hop increased about $35 \%$, compared with DV-Hop. 


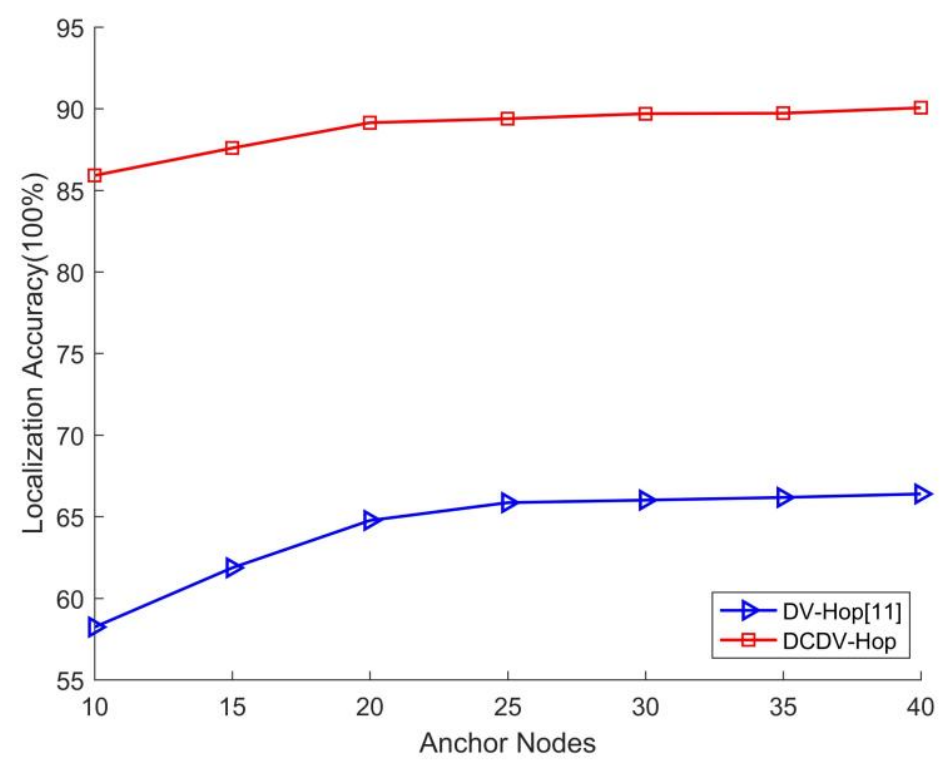

Fig. 10.The LA under various anchor nodes

Table 3. The comparison of ALE and LA for various anchor nodes

\begin{tabular}{|l|c|c|c|c|}
\hline \multicolumn{1}{|c|}{ Algorithm } & Min.ALE(m) & Avg.ALE(m) & Max.LA (100\%) & Avg.LA (100\%) \\
\hline DV-Hop & 8.53 & 9.03 & 65.89 & 63.86 \\
\hline DCDV-Hop & 4.21 & 4.41 & 90.06 & 88.37 \\
\hline
\end{tabular}

It is observed form Table 3, our proposed algorithm has a superior performance, with lower localization error and high localization accuracy. Besides, the ALE of DCDV-Hop is round $4.5 \mathrm{~m}$, but ALE of DV-Hop is large than $8.5 \mathrm{~m}$. Furthermore, enhanced minimum ALE and average ALE of DCDV-Hop is reduced $50.64 \%$ and $51.16 \%$, compared with basic DV-Hop, respectively. The average LA of proposed DCDV-Hop is more than $88 \%$, that means it with higher localization accuracy, but average LA of DV-Hop is less than 64\%. The maximum LA and average LA of DCDV-Hop is increased $24.17 \%$ and $24.51 \%$, compared with DV-Hop, respectively.

3. Effect of communication range on algorithm: To illustrate the effect of communication range on algorithm, a simulation experiment is performed in this section. The communication range $\mathrm{R}$ is from $20 \mathrm{~m}$ to $36 \mathrm{~m}$, with fixed 2 increased, the total number of nodes is 100 and anchor node ratio is $20 \%$. The ALE and LA under different communication ranges is represented in Fig. 11 and Fig.12 The maximum ALE and LA, average ALE and LA, minimum ALE and LA are demonstrated in Table 4. 
Paper-A Hybrid Range-Free Algorithm Using Dynamic Communication Range for Wireless Sens...

Table 4. The comparison of ALE and LA for various communication ranges

\begin{tabular}{|l|c|c|c|c|}
\hline \multicolumn{1}{|c|}{ Algorithm } & Min.ALE(m) & Avg.ALE (m) & Max.LA (100\%) & Avg.LA (100\%) \\
\hline DV-Hop & 7.21 & 8.39 & 69.34 & 63.98 \\
\hline DCDV-Hop & 3.55 & 5.09 & 90.94 & 89.31 \\
\hline
\end{tabular}

It is presented that in Table 4 our proposed algorithm has outstanding performance, with lower localization error and high localization accuracy. Besides, the ALA of DCDV-Hop is 5m, however, the ALE of DV-Hop is large than $7.2 \mathrm{~m}$. In addition, the enhanced minimum ALE and average ALE of DCDV-Hop is reduced 50.76\% and $39.33 \%$, compared with basic DV-Hop, respectively. The average LA of proposed DCDV-Hop is more than $88 \%$, that means it with higher localization accuracy, yet, maximum LA of DV-Hop is less than $70 \%$. The maximum LA and average LA of DCDV-Hop is increased $21.60 \%$ and $25.33 \%$, compared with DV-Hop, respectively.

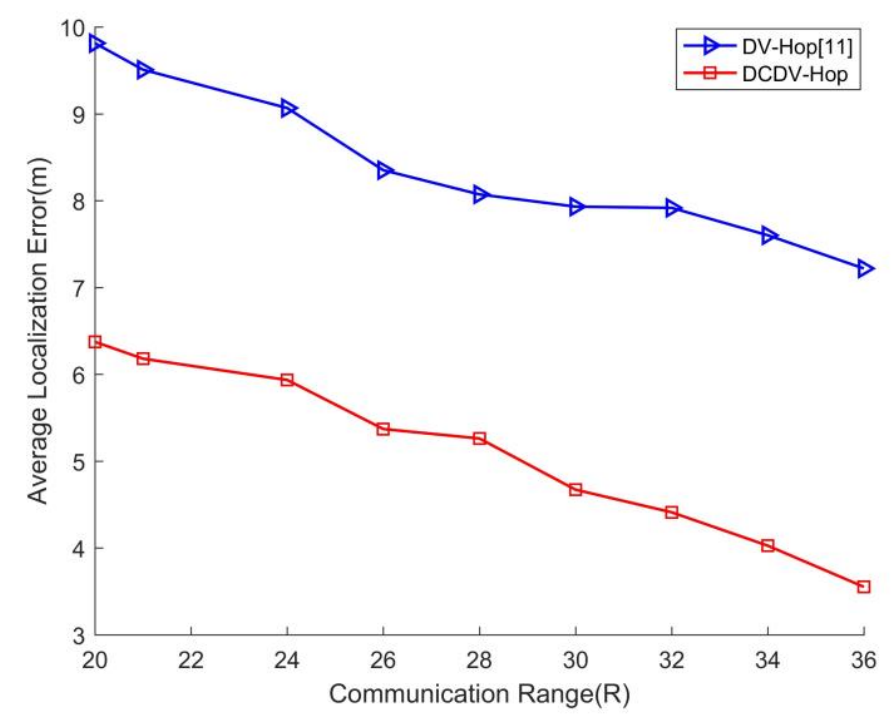

Fig. 11.The ALE under various communication ranges

As it can be seen from Fig.11, the ALE presented downward trend with the communication range increasing. If communication range is small, it is easy to cause the uneven network topology. Besides, with node communication range is increasing, the network connectivity will be enhanced, thereby, network topology will become regular. In addition, this will automatically reduce average localization error. The ALE of DCDV-Hop is less than $6.5 \mathrm{~m}$, with decreases more than $50 \%$, compared with DVHop algorithm. 


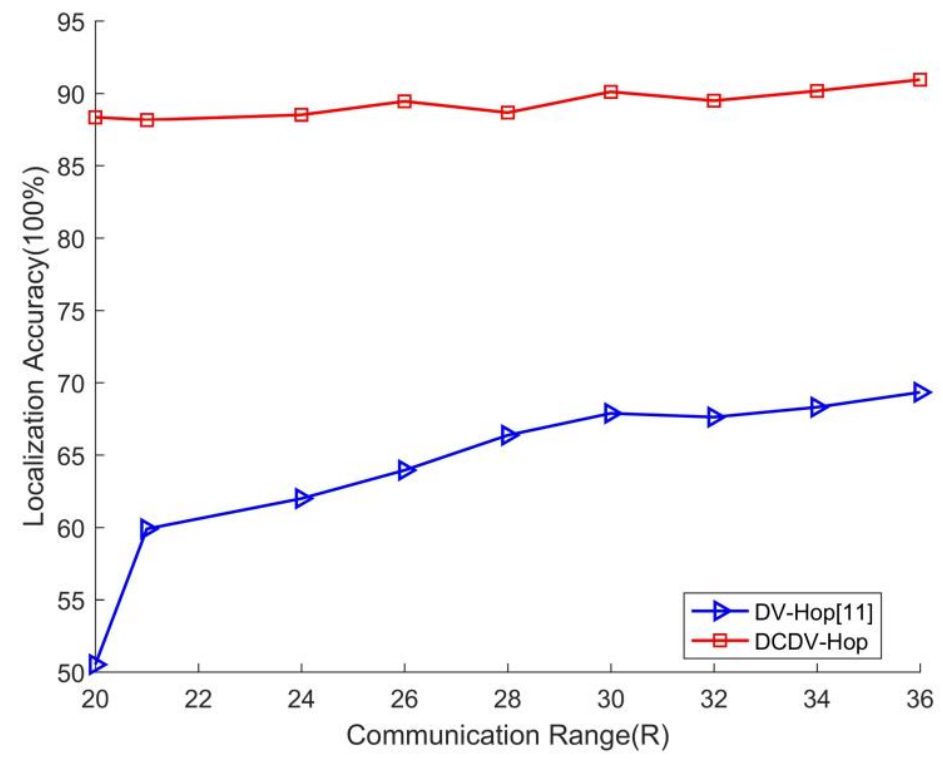

Fig. 12.The LA under various communication ranges

The LA is illustrated Fig.12. The both algorithms demonstrates upward trend with communication range increasing. The LA of our proposed DCDV-Hop is large than $85 \%$, however, the maximum LA is less than $70 \%$, which presents DCDV-Hop superior outstand than DV-Hop. The LA of DCDV-Hop is increased more than $30 \%$, compared with DV-Hop.

\section{Conclusion}

DV-Hop algorithm suffered poor localization accuracy due to one average hop size cannot represent for whole networks, since nodes are randomly deployed in monitoring area. The average hop size is directly decided by hop counts and communication ranges after deep analysis. Accordingly, we applied statistical methods to analyze the relationship between location error and hop count under different communication ranges. This paper proposed a new approach to optimize best hop count threshold based on our analyzed experiments. Moreover, enhanced centroid algorithm and weighted least square were employed to further correct target coordinate. We conducted extensive experiments under several effected parameters to evaluate our presented DCDV-Hop algorithm. It is verified that our proposed DCDV-Hop has shown superior performance, its localization error decreased more than 50\%, compared with DV-Hop algorithm. Besides, the maximum localization accuracy has reached up to $90.94 \%$. Our experiment only conducted in 2D, the next stage of our research will be implemented this idea in 3D WSNs and involve some environment factors. 
Paper-A Hybrid Range-Free Algorithm Using Dynamic Communication Range for Wireless Sens...

\section{$7 \quad$ Acknowledgement}

This research work was supported by Universiti Malaysia Pahang Postgraduate Research Grants, grant number is PGRS1903143.

\section{References}

[1] Akyildiz, I.F., Su, W., Sankarasubramaniam, Y., and Cayirci, E.J.I.C.m., 'A Survey on Sensor Networks', 2002, 40, (8), pp. 102-114. https://doi.org/10.1109/mcom.2002. $\underline{1024422}$

[2] Chowdhury, T.J., Elkin, C., Devabhaktuni, V., Rawat, D.B., and Oluoch, J.J.C.N., 'Advances on Localization Techniques for Wireless Sensor Networks: A Survey', 2016, 110, pp. 284-305. https://doi.org/10.1016/j.comnet.2016.10.006

[3] Suo, H., Wan, J., Huang, L., and Zou, C., 'Issues and Challenges of Wireless Sensor Networks Localization in Emerging Applications', in, 2012 International Conference on Computer Science and Electronics Engineering, (IEEE, 2012). https://doi.org/10.1109/ iccsee.2012.44

[4] Laoudias, C., Moreira, A., Kim, S., Lee, S., Wirola, L., Fischione, C.J.I.C.S., and Tutorials, 'A Survey of Enabling Technologies for Network Localization, Tracking, and Navigation', 2018, 20, (4), pp. 3607-3644. https://doi.org/10.1109/comst.2018.2855063

[5] Manickam, M., Selvaraj, S.J.I.S., Measurement, and Technology, 'Range-Based Localisation of a Wireless Sensor Network Using Jaya Algorithm', 2019. https://doi.org/10. 1049/iet-smt.2018.5225

[6] Harter, A., Hopper, A., Steggles, P., Ward, A., and Webster, P.J.W.N., 'The Anatomy of a Context-Aware Application', 2002, 8, (2/3), pp. 187-197.

[7] Girod, L., Bychkovskiy, V., Elson, J., and Estrin, D., 'Locating Tiny Sensors in Time and Space: A Case Study', in, Proceedings. IEEE International Conference on Computer Design: VLSI in Computers and Processors, (IEEE, 2002). https://doi.org/10.1109/iccd. 2002.1106773

[8] Bulusu, N., Heidemann, J., and Estrin, D.J.I.p.c., 'Gps-Less Low-Cost Outdoor Localization for Very Small Devices', 2000, 7, (5), pp. 28-34. https://doi.org/10.1109/98.878 $\underline{533}$

[9] Zhao, L.-z., Wen, X.-b., and Li, D., 'Amorphous Localization Algorithm Based on Bp Artificial Neural Network', 2014.

[10] Kulkarni, V.R., Desai, V., and Kulkarni, R.V.J.W.N., 'A Comparative Investigation of Deterministic and Metaheuristic Algorithms for Node Localization in Wireless Sensor Networks', 2019, 25, (5), pp. 2789-2803. https://doi.org/10.1007/s11276-019-01994-9

[11] Niculescu, D. and Nath, B.J.T.S., 'Dv Based Positioning in Ad Hoc Networks', 2003, 22, (1-4), pp. 267-280.

[12] He, T., Huang, C., Blum, B.M., Stankovic, J.A., and Abdelzaher, T., 'Range-Free Localization Schemes for Large Scale Sensor Networks', in, Proceedings of the 9th annual international conference on Mobile computing and networking, (ACM, 2003). https://doi.org/10.1145/938985.938995

[13] Shi, H. and Peng, L., 'An Improved Dv-Hop Node Localization Algorithm Combined with Rssi Ranging Technology', in, Proceedings of the 5th International Conference on Electrical Engineering and Automatic Control, (Springer, 2016). https://doi.org/10. 1007/978-3-662-48768-6_31 
[14] Huang, C., Mao, Y., ' Exploration of a New Location Algorithm for Wireless Sensor Network', 2018, 14 (06), pp.191-202

[15] Wu, J., 'The Research and Optimization of Ad-Hoc Network Localization Algorithm Based on the Distance-Assisted', in, 2016 18th International Conference on Advanced Communication Technology (ICACT), (IEEE). https://doi.org/10.1109/icact.2016. $\underline{7423446}$

[16] Hu, D., Liu, G., and Li, H., 'Study on Wireless Sensor Networks Localization Algorithm Based on Dv-Hop', in, 2016 2nd Workshop on Advanced Research and Technology in Industry Applications (WARTIA-16), (Atlantis Press, 2016). https://doi.org/10.2991/wartia$\underline{16.2016 .194}$

[17] Li, Y.-H., Wu, S.-H., and Wu, H.-T., 'A Design of Wireless Sensor Network Node Location Algorithm', in, 2016 International Conference on Computer and Information Technology Applications, (Atlantis Press, 2016). https://doi.org/10.2991/iccita-16.2016.18

[18] Salama, M. and Kandil, M., 'An Improved Dv-Hop Localization Algorithm Based on Modified Hop-Size', in, 2016 World Symposium on Computer Applications \& Research (WSCAR), (IEEE, 2016). https://doi.org/10.1109/wscar.2016.11

[19] Zheng, J.-g., Chen, L., and Xia, X.-h., 'The Localization Algorithm Based on Improved Dv-Hop Algorithm', in, 2nd International Conference on Electronics, Network and Computer Engineering (ICENCE 2016), (Atlantis Press, 2016). https://doi.org/10.2991/icence$\underline{16.2016 .83}$

[20] Tomic, S. and Mezei, I.J.T.S., 'Improvements of Dv-Hop Localization Algorithm for Wireless Sensor Networks', 2016, 61, (1), pp. 93-106. https://doi.org/10.1007/s11235-015$\underline{0014-9}$

[21] Xiong, N.N., Wu, W., Wu, C., and Cheng, H.J.J.o.I.T., 'An Improved Node Localization Algorithm Based on Positioning Accurately in Wsn', 2019, 20, (5), pp. 1323-1332.

[22] Cao, Y. and Wang, Z.J.I.A., 'Improved Dv-Hop Localization Algorithm Based on Dynamic Anchor Node Set for Wireless Sensor Networks', 2019, 7, pp. 124876-124890. https://doi.org/10.1109/access.2019.2938558

[23] Liu, Y, Yu, X, Hao, Y', Wireless Sensor Node Localization Algorithm Based on Particle Swarm Optimization and Quantum Neural Network', 2018, 14 (10), pp.230-240. https://doi.org/10.3991/ijoe.v14i10.9314

[24] Qiao, X., Yang, H., Wang Z..', Iterative L-M Algorithm in WSN - Utilizing Modifying Average Hopping Distances ', 2017, 13 (10), pp.4-20. https://doi.org/10.3991/ijoe. v13i10.7006

[25] Zheng, J, Wang, G, Chen, Y, Huang D, Yuan H, ' On the Localization Algorithm of Wireless Sensor Network and Its Application ', 2017, 13 (3), pp.40-51.

[26] Yan, X, Sun, L, Zhou, J, and Song, A.J.E.L, 'Dv-Hop Localisation Algorithm Based on Optimal Weighted Least Square in Irregular Areas', 2018, 54, (21), pp. 1243-1245. https://doi.org/10.1049/el.2018.6512

\section{Authors}

Fengrong Han is currently pursuing PHD degree program in faculty of electrical and electronics engineering, University Malaysia Pahang, Malaysia. E-mail: hfr825@163.com

Izzeldin Ibrahim Mohamed Abdelaziz is a senior lecture of faculty of electrical and electronics engineering at University Malaysia Pahang, Malaysia. E-mail: izzeldin@ump.edu.my 
Paper-A Hybrid Range-Free Algorithm Using Dynamic Communication Range for Wireless Sens..

Xinni Liu is currently pursuing PHD degree program in faculty of electrical and electronics engineering, University Malaysia Pahang, Malaysia. E-mail: PEG18006@stdmail.ump.edu.my

Kamarul Hawari Ghazali is a Professor of faculty of electrical and electronics engineering at University Malaysia Pahang, Malaysia. He is a senior member IEEE and a member of IEEE communications society chapter. E-mail: kamarul@ump.edu.my

Hao Wang is currently pursuing PHD degree program in Faculty of Manufacturing and Mechatronic Engineering Technology, University Malaysia Pahang. E-mail: PFD19002@stdmail.ump.edu.my

Article submitted 2020-03-24. Resubmitted 2020-04-10. Final acceptance 2020-04-12. Final version published as submitted by the authors. 